\title{
Study Response of Turbulence in Transition of Unsteady Channel Flows
}

\author{
B. S. Oluwadare, S. He \\ Department of Mechanical Engineering, University of Sheffield \\ Sheffield, S1 3JD, United Kingdom \\ bsoluwadare1@sheffield.ac.uk; s.he@ sheffield.ac.uk
}

\begin{abstract}
Experimental study is carried out to study the response of turbulence in an unsteady turbulent flow subject to a rapid increase of flow rate. The study confirms the concept proposed by He and Seddighi [1] (J Fluid Mech 715:60-102, 2013) based on DNS results that transient channel flow undergoes a process of transition that resembles the laminar-turbulent bypass transition despite the initial flow is turbulent. Particle Image Velocimetry (PIV) is used to obtain the instantaneous velocity of the unsteady flows. The determination of the skin friction coefficient of the unsteady flows is obtained from hot-film anemometry. A predetermined flow variation is obtained through a pneumatically regulated valve. During the acceleration of the channel flow, the Reynolds number is increased rapidly from the initial Reynolds number to the final Reynolds number. In response to the rapid increase of flow rate, the skin friction coefficient increases sharply resulting from the creation of a thin boundary layer of high strain near to the wall. As the boundary layer thickness increases, the viscous force reduces, and skin friction coefficient decreases to a minimal point that marks the onset of transition. During the transitional period, new turbulence structures are generated that make the skin friction coefficient to increase again. The present study conforms with the findings of the previous DNS that the transient flow involves three phases, namely pre-transition, transition and fully turbulent. The three distinct phases are equivalent to the three regions of boundary layer bypass transition which are buffeted laminar flow, intermittent flow, and fully turbulent flow regions and the flow characteristics are similar to previous numerical results on channel transient flows.
\end{abstract}

Keywords: Unsteady Channel Flow, Turbulence, Transition, DNS, PIV, CTA.

\section{Introduction}

The study of unsteady flow to find solutions to engineering problems has been on-going for over a century. Unsteady flow occurs in many applications of engineering: pipeline networks that contain reciprocating pumps in oil and gas industries, internal combustion engines, nuclear power plants, and pneumatic control systems. It also occurs in natural systems such as the flow of blood in arteries. Understanding the turbulence characteristics of unsteady-mean turbulent flows will help the design of the systems. In engineering design problems, the transition to turbulence occurs, and a good understanding of the transitional flow characteristics will help in controlling the problems. Transition of laminar flow to turbulent has been studied by numerous researchers using numerical, theoretical and experimental techniques since the pioneering work carried out by Osborne Reynolds [2]. The significance of the knowledge of laminar-turbulent transition has motivated numerous investigators in the area of fluid mechanics. The knowledge of the procedures involved in the transitional flow has a significant impact in proffering solutions to many engineering problems such as the management of wall shear stress, mixing processes, flow prediction and heat transfer in engineering. In flat boundary layers, there are two classes of transition to turbulence. The first class is natural transition and the second class of transition to turbulence is bypass transition. In a natural transition, the flow undergoes very slow changes due to viscous process, and the two-dimensional Tollmien-Schlichting (TS) waves are generated and the changes (primary TS waves) develop to a three-dimensional secondary instability and consequently, break down to turbulent flows. The free-stream turbulence in natural transition is far less than $0.01(T u \ll 1 \%)$. The flow is characterized by a slow process and occurs at high transitional Reynolds number, $R e_{x}\left(=\frac{x U_{\infty}}{v}=10^{6}\right)$ which is based on the free-stream velocity and the distance from the leading edge (Kachanov [3]; Kleiser \& Zang [4]). In the second class of transition, which is known as bypass transition, the level of free-stream turbulence or turbulence intensity $(T u)$ is greater than $0.01(\mathrm{Tu}>1 \%)$; however, the breakdown to turbulence takes place at the transitional Reynolds number which is less than or equal to $\operatorname{Re}_{x}\left(=10^{5}\right)$. Under such conditions the disturbances in the flow quickly develop, and the transition to turbulence occurs promptly, and Tollmien-Schlichting waves are bypassed. The process is called bypass transition (Jacobs \& Durbin [5]). Schubauer and Skramstad [6] were the first to recognize that natural TollmienSchlichting waves are induced by free stream turbulence by placing a rope grid in the settling chamber of the wind 
tunnel, which reveals the wave activity and hastens the development of pre-transitional structures and turbulent spots. The experimental results were used to verify the solutions for unstable waves travel in the streamwise direction of the flow presented by Tollmien [7] and Schlichting [8]. Boiko et al. [9] investigated the effects of Tollmien-Schlichting (TS) waves on the transition at free stream turbulence equal to $0.015(T u=1.5 \%)$ and reported that at smallamplitude, TS-waves interact nonlinearly, which results in a redevelopment of TS-waves in the fluctuating frequency band. The investigations carried out by Kendall [ 10] and Westin et al. [11] showed that the transition to turbulence in boundary layers subjected to free-stream turbulence takes place as a result of strong streamwise streaks. In a bypass transition process, the flow passes through three zones of flow development: the buffeted laminar boundary layer; intermittent turbulent spot formation and the fully turbulent boundary layer. The buffeted laminar boundary layer region is typified by the creation of thin boundary layer and the development of skin friction and streamwise velocity fluctuations near the wall. The amplitude of the disturbances increases in the streamwise direction leading to the elongation of the streaks. Low-frequency disturbances penetrate through the boundary layer resulting in elongated streaks, but the ones at high-frequency are filtrated out by the boundary layer (Jacobs and Durbin [5]). Kendall [10] studied the elongated structures in streamwise direction with small spanwise scales in a laminar boundary layer subjected to free-stream turbulence and referred the disturbances generated as Klebanoff modes (the formation of streaks). Klebanoff modes are someway believed to be responsible for the transition to turbulence, and numerous experimental investigations have been carried out to extensively study the modes (Westin et al. [11]; Matsubara \& Alfredsson [12]). In the region of intermittent turbulent spots formation, localized disturbances trigger the instabilities that finally change into turbulent spots which increase in size and merge with each other further downstream of the boundary layer flow. In the fully turbulent boundary layer region, the entire span of the boundary layer flow is covered by turbulence structures.

The new perceptive of the unsteady turbulent flow of He and Seddighi [1] is based on laminar-turbulent bypass transition, and the present study focuses on the expansion of the above study. Direct numerical simulations of He and Seddighi [1] have revealed that turbulence does not progressively evolve from one state to another in the flow, but the flow is characterized with the development of boundary layer and followed by a transition to turbulence even though the initial flow is turbulent. They reported that the transient procedure of a low-Reynolds-number turbulent flow resembles laminar-turbulent transition. In response to the sudden increase of flow rate, the flow undergoes three distinct stages of transition (pre-transition, transition and fully turbulent) that are equal to the three regions of boundary layer bypass transition (buffeted laminar boundary layer, intermittent turbulent spot formation, and the fully turbulent boundary layer). The bypass transition in the unsteady channel flow represents an alternative free stream turbulence (FST) induced transition. In this method of bypass transition, the required disturbance in the flow was provided by a low Reynolds number turbulent wall shear flow with pre-existing streaky structures. As the flow rate is increased suddenly, a new boundary layer is formed. When the flow rate increases, the flow changes from the earlier turbulent structure into a new one after passing through the three stages of transition mentioned above. The numerical results of slower accelerating turbulent flow of Seddighi et al. [13] are similar to earlier DNS results reported by He \& Seddighi [1] It was revealed that both accelerating flow and step-increase of flow rate undergo three distinct phases of transition, but the stretching of the streaks in an accelerating flow during the pre-transition is not strong. The authors reported that a sharp boundary layer which closely resembles spatially developing boundary layer was generated over the wall following a step-increase of flow rate whereas gradual increase of the flow rate in an accelerating flow causes a gradually-developing boundary layer. The transition was much delayed in Seddighi et al. [13] compared to He \& Seddighi [1]. Contour plots in Figure1 shows the flow behaviour of the slower accelerating flow. The fluctuating velocity in streamwise direction normalized by initial bulk velocity was used to show the turbulence structure at a different period, although the changes that take place in accelerating flow are very small in comparison with the stepincrease of He \& Seddighi [1]. Between $t^{*}=0$ and $t^{*}=14$, that is the pre-transitional stage, streaks were formed, stretched and elongated. Between $t^{*}=18$ and $t^{*}=24$, known as the transitional stage, isolated turbulent spots were formed from the elongated streaks and spread and fill the wall-bounded surface. The fully turbulent stage occurred when the entire wall surface was covered with new turbulence structures. 

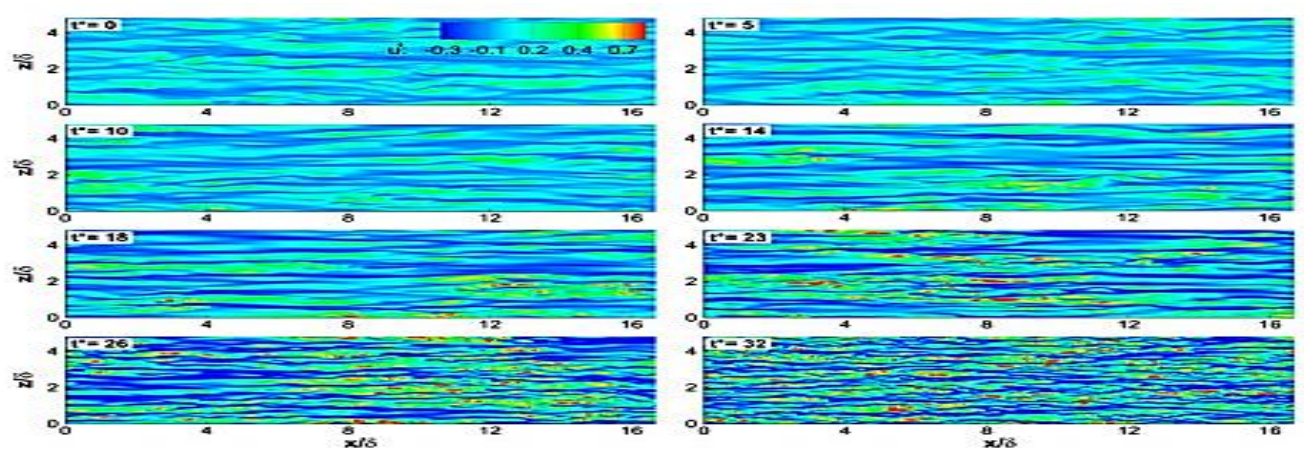

Fig. 1: Contour plots of streamwise fluctuations acceleration flow (Seddigh et al., [13]).

He and Seddighi [14] investigated the effects of free-stream turbulence on the transition of unsteady channel flow using direct numerical simulations. The Reynolds numbers (initial and final) of the unsteady flows were orderly varied to obtain different free-stream turbulence (FST) intensities. The effects of the free-stream turbulence structures due to the variation of initial and final Reynolds numbers were compared with the results of the transition induced by freestream turbulence in boundary layer flows. In boundary layer flow research, it has been found that the transitional Reynolds number is inversely proportional to the square of free-stream turbulence intensity $\left(R e_{c r} \sim T u^{-2}\right)$ (Fransson et al., 2005). The point at which transition occurs is expedient in the study of bypass transition in unsteady flow. When the friction coefficient $C_{f}\left(=\frac{\tau_{w}}{\frac{1}{2} \rho U_{b i}^{2}}=2\left(\frac{U_{\tau}^{2}}{U_{b 1}^{2}}\right)\right)$ is plotted against time $(t)$ or an equivalent Reynolds number $R e_{t}\left(=\frac{t U_{b 1}^{2}}{v}\right)$, the time at which minimum friction coefficient is recorded marks the beginning of the transition.

\section{Methodology}

In this section, the processes involved in the measurement of mean velocity and turbulent statistics are explained. All experiments were conducted using the channel flow facility in Figure 2. The experimental Test Rig is of length, width, and height of $8000 \mathrm{~mm}, 350 \mathrm{~mm}$ and $50 \mathrm{~mm}$ respectively and water is used as the working fluid. It also consists of the following components: bottom tank, overhead water tank, centrifugal pump, magnetic flow meter, pneumatically control valve, stainless steel adaptor, test section, measuring section and a honeycomb flow straightener. The height of the overhead water tank is located $5.5 \mathrm{~m}$ above the test section while the height of the wall of the channel to the ground is $1.21 \mathrm{~m}$.

A four-inch bore $2.2 \mathrm{KW}$, three-phase, four-pole centrifugal pump transfers water from the bottom tank through 4inches PVC transfer pipeline to the overhead water tank. The end of the transfer pipeline was covered with a mesh screen, to remove the bubbles that result from the air entrapment and cavitation from the bottom tank. Overflow pipeline transfers excess water from the overhead water tank to the bottom tank ensures the driving pressure gradient is achieved by maintaining a constant height of the overhead water tank throughout the experiments. Metallic-coated, hollow- glass spheres with mean diameter of $14 \mu \mathrm{m}$ and density of $1.65 \mathrm{~g} / \mathrm{cc}\left(1650 \mathrm{~kg} / \mathrm{m}^{3}\right)$ was added to the water in the bottom tank. The water from the overhead water tank flows through a manually regulated on/off valve on the PVC pipeline into the test section through the stainless-steel adaptor and honeycomb flow straightener that removes large swirling flow structures from the upstream.

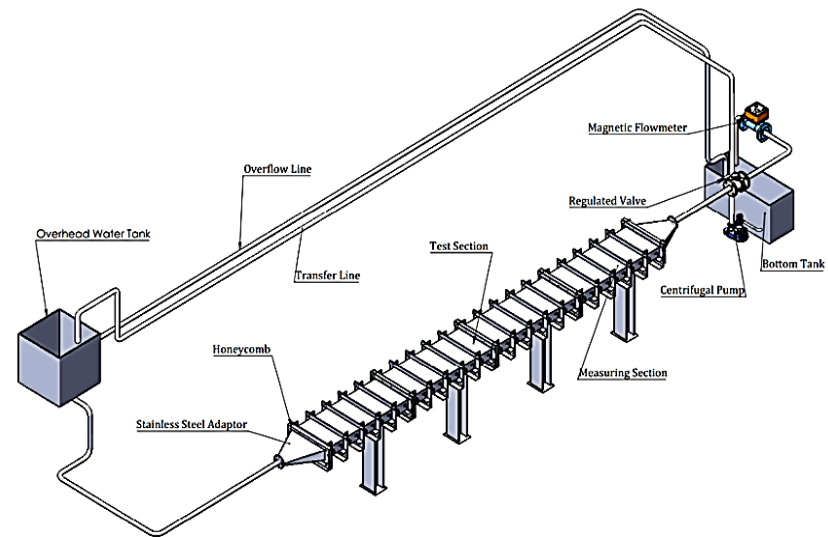

Fig. 2: Schematic diagram of the channel flow facility. 
It then flows through the test section, the stainless-steel adaptor passing through a pneumatically control valve and a magnetic flow meter before it is discharged into the bottom tank. The pipeline that extends from the magnetic flow meter is submerged properly into the bottom tank in order to reduce the formation of bubbles in the bottom tank. Transparent Perspex Plates are used for the construction of the test channel section. The test section consists of four compartments, and the length of each compartment is $2000 \mathrm{~mm}$. The measuring equipment-laser, camera, and hot-film sensor are mounted on the fourth compartment of the test section. In order to increase the optical access at the fourth compartment of the test section (measurement section), a glass window is installed onto one side of the measuring section. The measuring window is at $7000 \mathrm{~mm}$ from the flow straightener to ensure the spatially developed of the measured flow. Dantec dynamic PIV system that consists of a double pulse laser (Nd-YAG $65 \mathrm{~mJ} / \mathrm{pulse}$ ) with time between pulses of $400 \mu$ s or $600 \mu$ s and a 12-bit Charge-Coupled Device Camera of 2048 x 2048 pixels resolution with a frequency of $7 \mathrm{~Hz}$ were used to determine velocity vectors in the flow. The measurements of velocity field are obtained in two different camera-laser orientations. The first orientation enables the laser to fire from the top of the test channel (vertical-PIV) and the second orientation enables the laser to fire from the side of the test channel (horizontalPIV). These orientations allow measurements of the velocity vectors in both horizontal and vertical planes. The hotfilm sensors panel is installed at a distance of $7200 \mathrm{~mm}$ from the inlet of the test section and is used to measure wall shear stress at sampling rates of $7 \mathrm{~Hz}$. The required transient flows were generated by quickly opening the pneumatically control valve that is located at the downstream of the test section. Dantec dynamic software is used to calculate the velocity field from the displacement of seeding particles and the known values of the time difference between the pulses. Images from both frames are divided into different interrogation areas and form a group of particles in each interrogation area. The group of particles in each interrogation area produces a unique thumbprint in the frame one. The unique thumbprint which is identified in the frame one should be traceable in frame two within the interrogation area. Cross-correlation is calculated at each location within the interrogation area. A maximum crosscorrelation is acquired as the thumbprint is identified in frame two. The required displacement vector is obtained when the thumbprint in frame two interrogation area is offset on the frame one. As the value of displacement is obtained, then the velocity is obtained by dividing the displacement by the time between pulses, and the procedure is repeated for all the remaining interrogation areas.

\section{Results and Discussion}

\subsection{Acceleration Flow}

Figure 3 presents the bulk velocity variation for cases U1 and U2 and Figures 6 (a) shows that of cases K1, K2, K3 and K4. The bulk velocity variation is attained when the control valve is opened suddenly from the initial Reynolds number to the final Reynolds number. The ensemble-average is performed on 120 realizations to obtain the transient flow development for all the cases. The acceleration time, $\Delta t$ is the time needed for the initial bulk velocity to attain $80 \%$ of the final flow. The flow

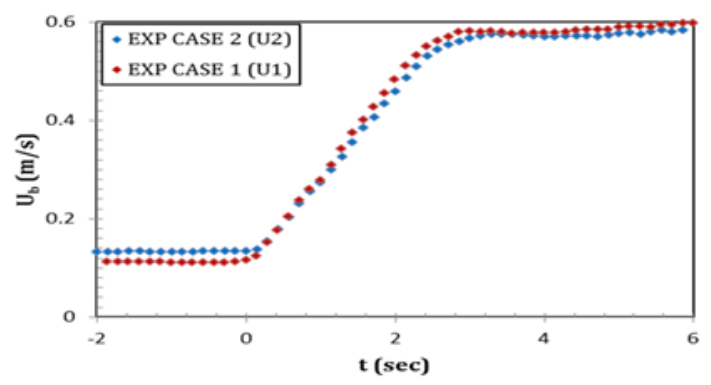

Fig. 3: Bulk velocity variation for case U1 (Reb =2800-15000) \& case U2 (Reb =3500-15000).

conditions of the present study are shown in Table 1 . The bulk Reynolds number is defined as $\operatorname{Re}_{b}=U_{b} \delta / v$ where $U_{b}$ is defined as the bulk velocity and $\delta$ is the channel half-height. The measurements of velocity field are obtained in two different camera-laser orientations. The first orientation enables the laser to fire from the top of the test section (termed vertical-PIV hereafter) and the second orientation enables the laser to fire from the side of the test section (termed parallel-PIV hereafter). Field of view (FOV) of $28.32 \mathrm{~mm} \times 28.32 \mathrm{~mm}$ and interrogation area (IA) of 32 pixels x 32 pixels are utilized during vertical-PIV camera-laser orientation. The instantaneous behaviour of the flow is obtained on parallel-PIV camera-laser orientation through the field of view (FOV) of $79 \mathrm{~mm} \times 79 \mathrm{~mm}$ and interrogation area (IA) of 32 pixels x 32 pixels. The time between pulses for both vertical-PIV camera-laser orientation and parallel-PIV 
camera-laser orientation is $400 \mu$ s and $600 \mu$ s respectively. The error of the present measurement technique is caused mainly by a drift in the Constant Temperature Anemometer calibration.

The experimental cases U1 and case U2 reported in this present study were compared with Mathur's [15] higher Reynolds number case E2. Mathur [15] reported the times when the transition begins and ends as 2.05 and 3.30 seconds respectively. Hot-film data of the present study are in close agreement with the Mathur's [15] data although the beginning of transition and end of the transition for the two cases occurred at different times see Figures 4(a) \& $5(\mathrm{a})$.

\subsection{Effect Skin Friction Coefficient, Mean And R.M.S Fluctuating Velocities in Transient Channel Flow}

Table 1: Times for onset transition and end of transition.

\begin{tabular}{|c|c|c|c|c|c|c|}
\hline & Case & $R e_{b 0}$ & $R e_{b 1}$ & $\Delta t(\mathrm{sec})$ & $t_{c r}(\mathrm{sec})$ & $t_{\text {turb }}(\mathrm{sec})$ \\
\hline Present & $\mathrm{U} 1$ & 2800 & 15000 & 2.00 & 2.60 & 3.50 \\
\hline Present & $\mathrm{U} 2$ & 3500 & 15000 & 2.15 & 2.10 & 3.10 \\
\hline Present & $\mathrm{K} 1$ & 2800 & 15000 & 1.72 & 2.09 & 3.08 \\
\hline Present & $\mathrm{K} 2$ & 2800 & 15000 & 1.88 & 2.37 & 3.32 \\
\hline Present & K3 & 2800 & 15000 & 2.37 & 2.89 & 4.36 \\
\hline Present & K4 & 2800 & 15000 & 3.42 & 3.40 & 5.09 \\
\hline Present & K5 & 2800 & 15000 & 5.24 & 3.93 & 6.23 \\
\hline $\begin{array}{c}\text { Mathur } \\
\text { (2016) }\end{array}$ & E2 & 2800 & 15500 & 1.90 & 2.05 & 3.30 \\
\hline
\end{tabular}

This present experimental study confirms the earlier results of DNS data of He \& Seddighi [1, 14] that the transition of transient channel flow resembles laminar-turbulent transition even though the initial flow condition is turbulent. Immediately when the flow excursion started, skin friction coefficient rises sharply, reaching the maximum value within a fraction of a second. This results from the creation of thin boundary layer near the wall that increases both viscous force and velocity gradient.

(a)

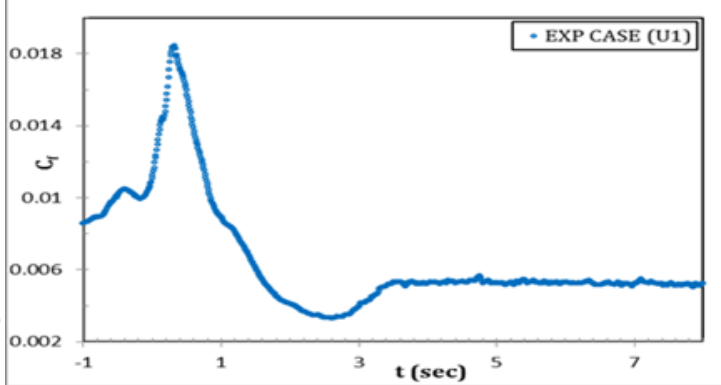

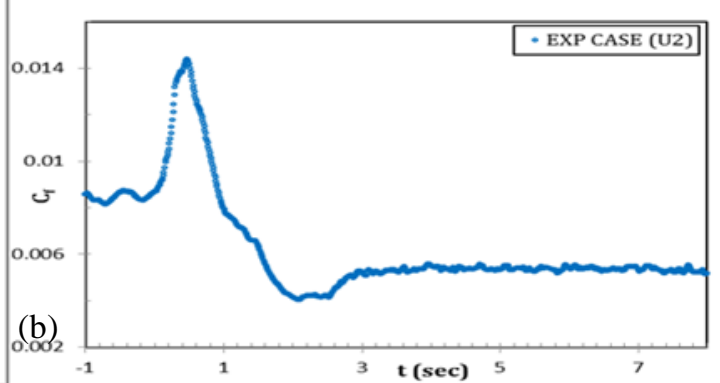

Fig. 4: Growth of the friction coefficient with time during the unsteady flow of case U1 $\left(\operatorname{Re}_{b}=2800-15000\right)$ and case U2 $\left(\operatorname{Re}_{\mathrm{b}}=3500-15000\right)$.
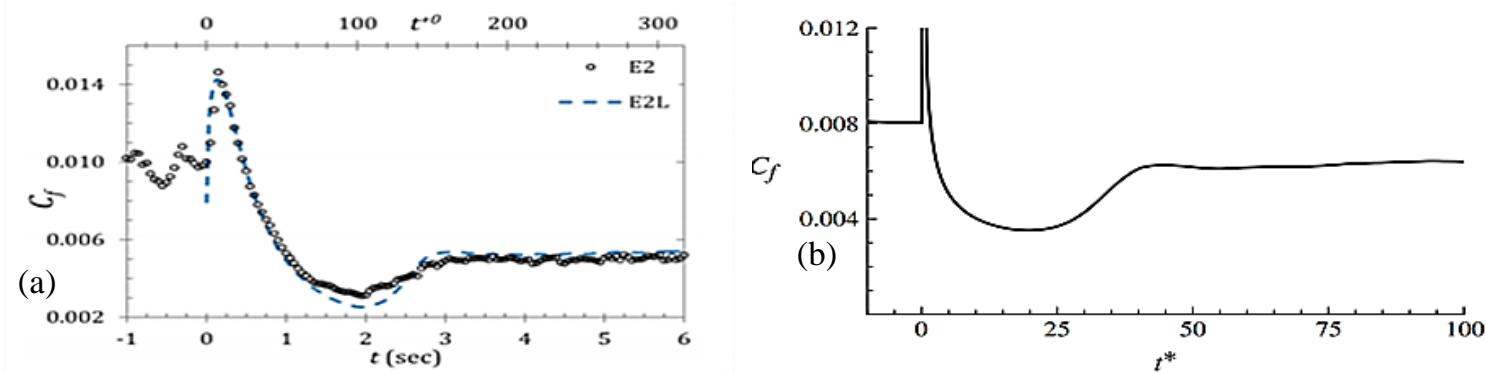

Fig. 5: Growth of the friction coefficient and wall shear stress with time during the unsteady flow of Mathur's [15] case E2 and He and Seddighi [1]. 

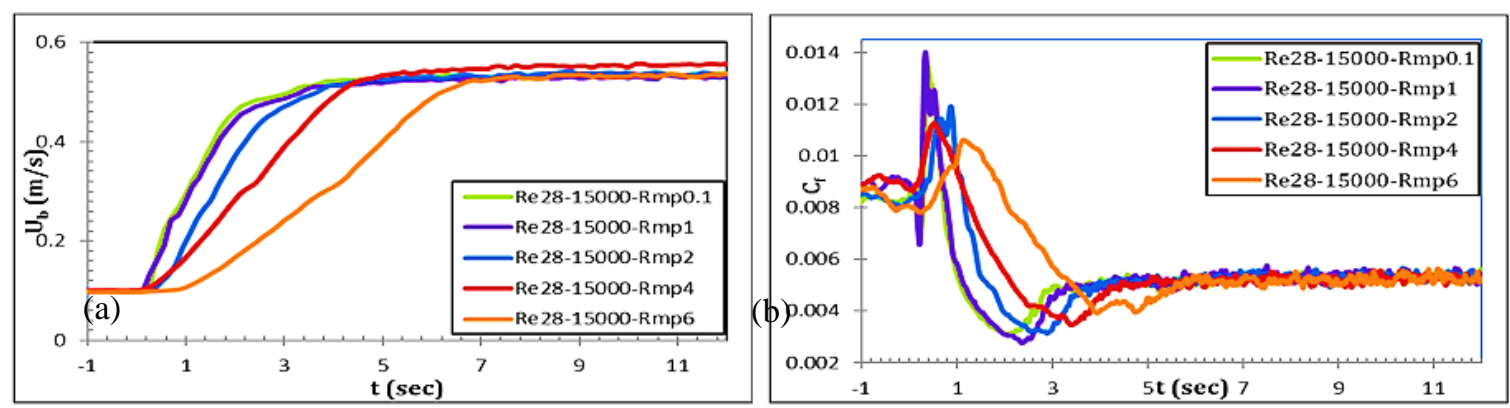

Fig. 6: Bulk velocity variation and growth of the friction coefficient with time during the unsteady flow of $\mathrm{Re}_{\mathrm{b}}=2800-15000$ with different acceleration times (cases K1, K2, K3, K4 and K5).

The growth of the thin boundary layer during the pre-transitional time frame causes the skin friction coefficient to reduce and reaching a minimum value at 2.60 and 2.10 seconds for case U1 and case U2 respectively as shown in Figures $4 \mathrm{a} \& \mathrm{bb}$. He and Seddighi [1] referred to the time at which the skin friction coefficient reached the minimum value as the critical time, which marks the beginning of the transition. The prompt increase of the skin friction coefficient is observed due to the creation of new
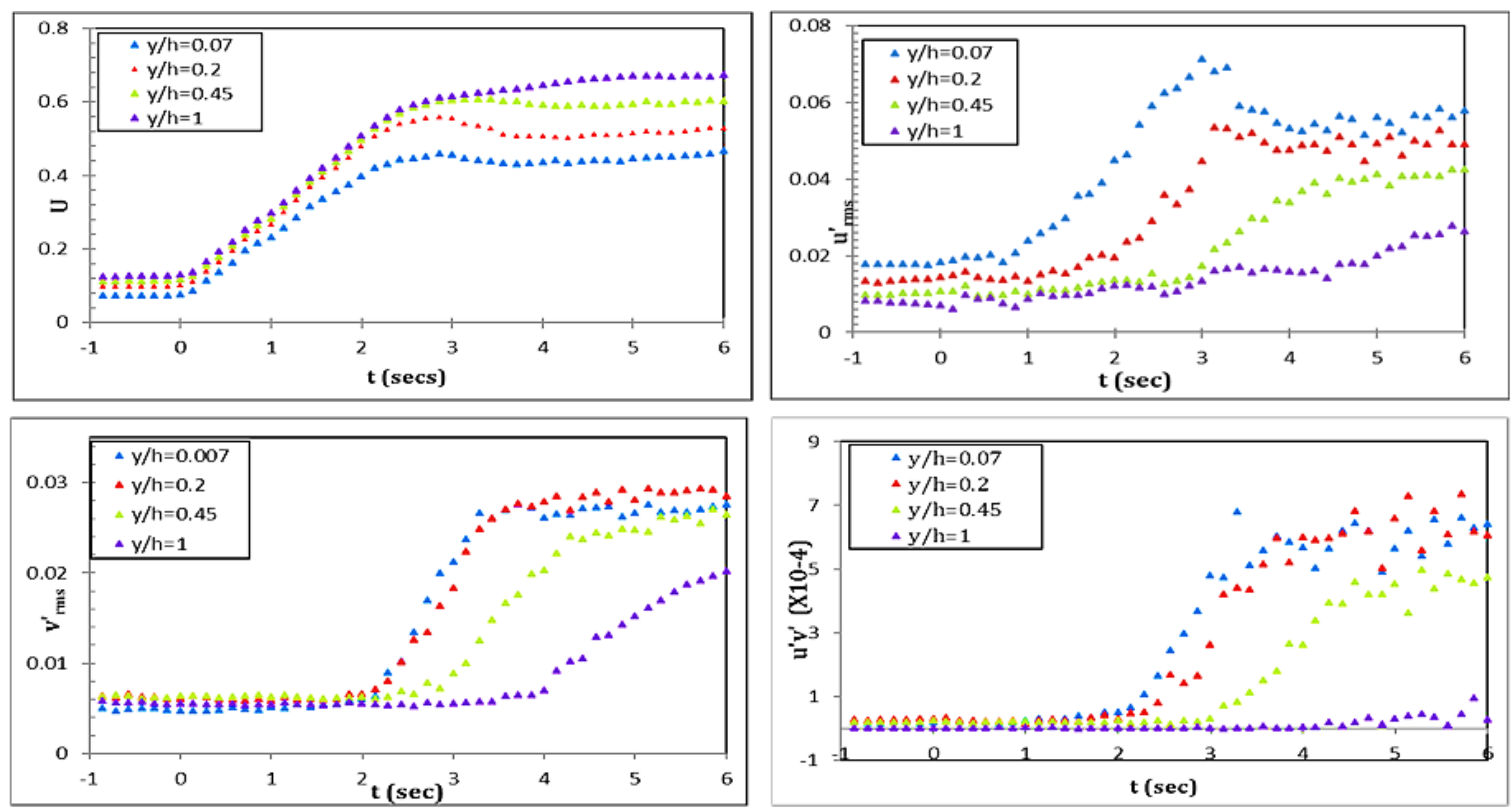

Fig. 6: Unsteady flow growth of Reb $=2800-15000$.

turbulence structures at the beginning of the transition. The skin friction coefficient increases to the final steady values marking the end of transition of case U1 and case U2 at 3.50 and 3.10 seconds respectively as shown in Figures 4a \& 4b. The times for onset and end of transition for cases K1, K2, K3, K4 and K5 are shown in Table 1. At the minimum value of skin friction coefficient, which is equivalent to critical time on the plot, turbulent spots are initially noted. When the transition is completed in the flow, newly produced isolated turbulent structures fill the entire wall-bounded surface, when the flow has fully become turbulent.

The behaviours of mean velocities and r.m.s fluctuating velocities are shown in Figure 6 for this present study. The required flow variation for the two cases is attained when the pneumatically regulated valve is opened suddenly from the initial to final flow conditions. Vertical-PIV laser-camera orientation is used to capture the data. 120 repeated runs are performed for each of the cases reported. Ensemble and spatial averages are performed on the data for the determination of mean and r.m.s fluctuating velocities. The growth of the unsteady flow is studied at different wall locations such as, $\mathrm{y} / \mathrm{h}=0.07,0.2,0.45$ and 1 and the results obtained are compared with Mathur's [15] unsteady flow growth. It is observed from Figure 6, when the pneumatically regulated valve is opened suddenly in the pre-transitional period, the mean velocity $(U)$ at different locations responded to the unsteady flow in a plug-like. The streamwise fluctuating velocity, $u_{r m s}^{\prime}$ responded to the unsteady flow due to the elongation of the initial streaky structures in the flow. These streaky structures in the flow act as background disturbances to the thin boundary layer that created near 
the wall. The disturbances created by these streaky structures are similar to disturbances created by free stream turbulence in boundary layer bypass transition. Frames 1 and 2 refer to the pre-transitional stage in Figure 7 . In the pretransitional stage, both wall normal fluctuating velocity, $v_{r m s}^{\prime}$ and turbulent wall shear stress, $\overline{u^{\prime} v^{\prime}}$ do not respond to the flow as shown in Figure 6. During the transitional stage, wall normal fluctuating velocity and turbulent wall shear stress responded to flow and marked the onset of transition as shown in Figure 6. In the transitional stage, isolated turbulent spots are formed from the elongated streaks and spread between frames 3 and 4 in Figure 7 . At the last stage, turbulent spots covered the entire wall-bounded surface when the flow has fully become turbulent as shown from frame 5 in Figure 7. The trends of skin friction coefficient of $\mathrm{K} 1, \mathrm{~K} 2, \mathrm{~K} 3, \mathrm{~K} 4$, and $\mathrm{K} 5$ are similar to the cases $\mathrm{U} 1$, U2 and that Mathur's [15] case E2. The results show that an in accelerating time leads to a reduction in the time of onset transition for all the cases shown in Figure 6 (b). These findings conform with the slow acceleration of Seddighi et al. [13].
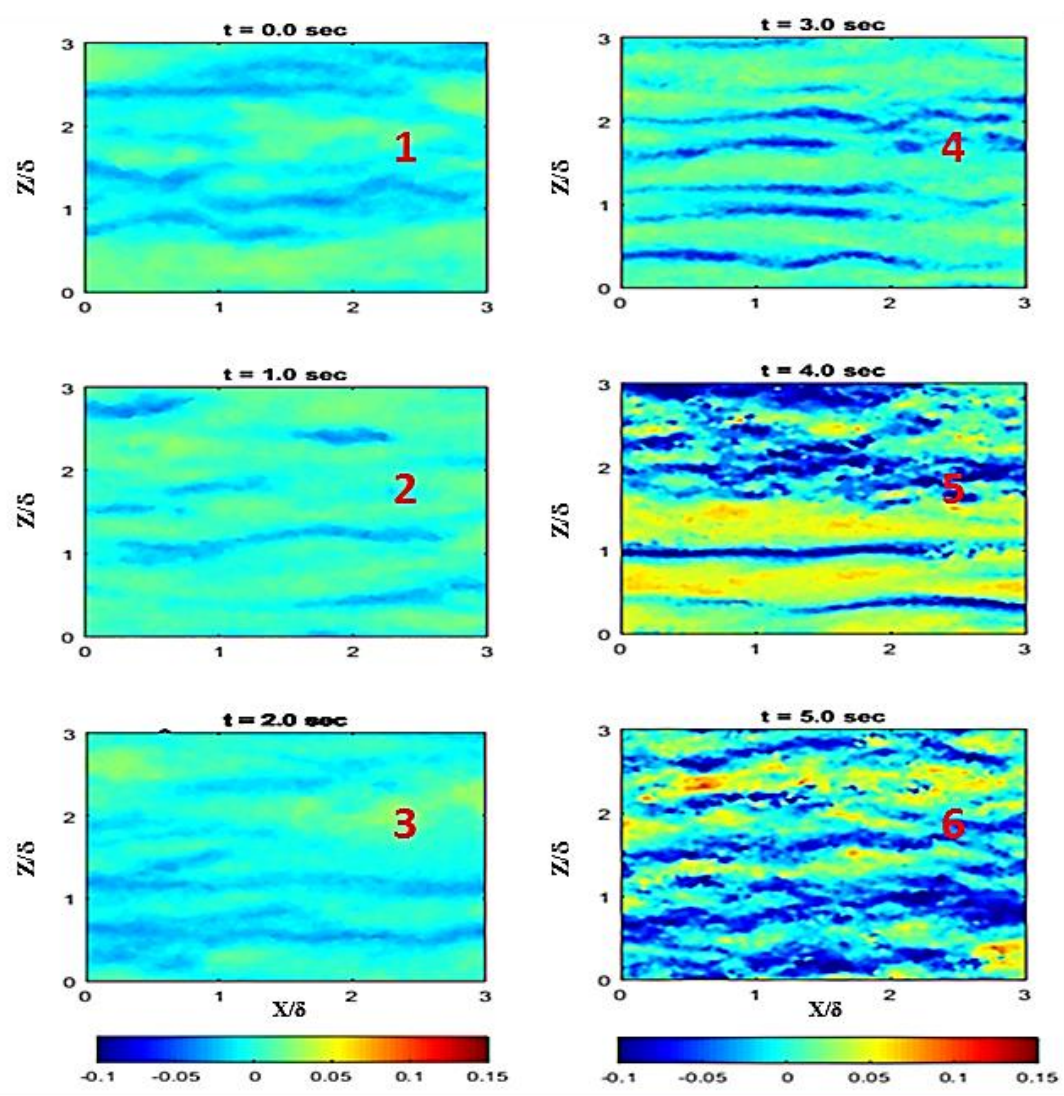

Fig. 7: Contour plots at a distance of $2 \mathrm{~mm}$ from the channel bed showing streamwise fluctuating velocity, $\boldsymbol{u}^{\prime}$ ( $\boldsymbol{m} / \boldsymbol{s}$ ) for unsteady flows at different time frames for case $\mathrm{U} 1\left(\mathrm{Re}_{\mathrm{b}}=2800-15000\right)$.

\section{Conclusion}

The new perspective of transient turbulent flows has been investigated experimentally using PIV and hot-film methods. It is revealed that turbulence does not progressively evolve from one state to another, but the flow is characterized by the development of a laminar-like boundary layer, followed by transition to turbulence. The process of transient channel flow that is subjected to a rapid increase in flow rate is a bypass transition process even though the initial flow condition is turbulent. It has three regions, namely pre-transition, transition and fully turbulent regions which are equivalent to the three regions of boundary layer bypass transition which are the buffeted laminar flow, the intermittent flow and the fully turbulent flow regions (for instance Jacob \& Durbin [5]). The processes of transition to turbulence reported are similar to earlier processes reported by numerical studies of He and Seddighi [1, 14], Seddighi, et al. [13] and that of Mathur [15].

\section{References}

[1] S. He and M. Seddighi, "Turbulence in transient channel flow," Journal of Fluid Mechanics, vol. 715, pp. 60$102,2013$. 
[2] O. Reynolds, "An experimental investigation of the circumstances which determine whether the motion of water shall be direct or sinuous, and of the law of resistance in parallel channels," Phil. Trans. R. Soc. Lond., vol. 174, pp. 935-982, 1883.

[3] Y. S. Kachanov, "Physical mechanisms of laminar-boundary-layer transition," Annual review of fluid mechanics, vol. 26, pp. 411-482, 1994.

[4] L. Kleiser and T. A. Zang, "Numerical simulation of transition in wall-bounded shear flows," Annual review of fluid mechanics, vol. 23, pp. 495-537, 1991.

[5] R. Jacobs and P. Durbin, "Simulations of bypass transition," Journal of Fluid Mechanics, vol. 428, pp. 185-212, 2001 .

[6] G. B. Schubauer and H. K. Skramstad, "Laminar boundary layer oscillations and transition on a flat plate," NACA Rep. 909, 1948.

[7] W. Tollmien, "Über die Entstehung der Turbulenz, Nachr. Ges. Wiss. Göttingen 21-24 (1929)," English translation NACA TM, 609, 1931.

[8] H. Schlichting, "Berechnung der Anfachung kleiner Störungen bei der Plattenströmung," English ZAMM., vol. 13, pp. 171-174, 1933.

[9] A. Boiko, K. Westin, B. Klingmann, V. Kozlov and P. Alfredsson, "Experiments in a boundary layer subjected to free stream turbulence. Part 2. The role of TS-waves in the transition process," Journal of Fluid Mechanics, vol. 218, pp. 219-245, 1994.

[10] J. Kendall, "Experimental study of disturbances produced in a pre-transitional laminar boundary layer by weak freestream turbulence," in Proceedings of the AIAA 18th International Conference on Fluid Dynamics and Plasmadynamics and Lasers, Cincinnati, Ohio, pp. 85-1695, 1985.

[11] K. Westin, A. Boiko, B. Klingmann, V. Kozlov and P. Alfredsson, "Experiments in a boundary layer subjected to free stream turbulence. Part 1. Boundary layer structure and receptivity," Journal of Fluid Mechanics, vol. 218, pp. 193-218, 1994.

[12] M. Mastsubara and P. H. Alfredsson, "Disturbance growth in boundary layers subjected to free-stream turbulence," Journal of Fluid Mechanics, vol. 430, pp. 149-168, 2001.

[13] M. Seddighi, S. He, A. Vardy and P. Orlandi, "Direct numerical simulation of an accelerating channel flow," Flow, Turbulence and Combustion, vol. 92, pp. 473-502, 2014.

[14] S. He and M. Seddighi, "Transition of transient channel flow after a change in Reynolds number," Journal of Fluid Mechanics, vol. 764, pp. 395-427, 2015.

[15] A. Mathur, "Study of accelerating and decelerating turbulent flows in a channel," Ph.D. dissertation, Dept. Mech. Eng., University of Sheffield. 